\title{
Ankylosis homologue (ANKH) controls extracellular citrate and pyrophosphate homeostasis and affects bone mechanical performance
}

Flora Szeri ${ }^{1}$, Stefan Lundkvist ${ }^{1}$, Sylvia Donnelly ${ }^{1}$, Udo F.H. Engelke ${ }^{2}$, Kyu Rhee ${ }^{3}$, Charlene Williams $^{4}$, John P. Sundberg ${ }^{5}$, Ron A. Wevers ${ }^{2}$, Ryan E. Tomlinson ${ }^{6}$, Robert Jansen ${ }^{3}$ and Koen van de Wetering ${ }^{1^{*}}$

${ }^{1}$ Department of Dermatology and Cutaneous Biology, Jefferson Institute of Molecular Medicine and PXE International Center of Excellence in Research and Clinical Care, Sidney Kimmel Medical College, Thomas Jefferson University, Philadelphia (PA), USA

${ }^{2}$ Translational Metabolic Laboratory, Department Laboratory Medicine, Radboud University Medical Centre, Nijmegen, The Netherlands.

${ }^{3}$ Division of Infectious Diseases, Department of Medicine, Weill Cornell Medicine, New York (NY), USA.

${ }^{4}$ Cooper Medical School of Rowan University, Glassboro (NJ), USA

${ }^{5}$ The Jackson Laboratory, Bar Harbor (ME), USA

${ }^{6}$ Department of Orthopaedic Surgery, Thomas Jefferson University, Philadelphia (PA), USA

\section{${ }^{*}$ Corresponding author:}

Koen van de Wetering, Department of Dermatology and Cutaneous Biology, Jefferson Institute of Molecular Medicine and PXE International Center of Excellence in Research and Clinical Care, Sidney Kimmel Medical College, Thomas Jefferson University, Philadelphia (PA), USA. Koen.vandeWetering@jefferson.edu. Tel: +1(215)503-5701

\section{Keywords:}

extracellular citrate, extracellular pyrophosphate, ATP release, ANKH/ANK, ectopic mineralization, bone 


\section{Abstract}

39 The membrane protein Ankylosis homologue (ANKH, mouse orthologue: ANK) prevents 40 mineralization of joint-space and articular cartilage. The accepted view is that ANKH mediates

41 cellular release of inorganic pyrophosphate (PPi), a strong physiological inhibitor of

42 mineralization. Using global metabolite profiling, we identified citrate as the most prominent

43 metabolite leaving HEK293 cells in an ANKH-dependent manner. Although PPi levels were

44 increased in culture medium of HEK293-ANKH cells, PPi was formed extracellularly after release

45 of ATP and other nucleoside triphosphates. Ank ${ }^{\text {anklank }}$ mice, which lack functional ANK, had

46 substantially reduced concentrations of citrate in plasma and urine, while citrate was undetectable

47 in urine of a human patient lacking functional ANKH. Bone hydroxyapatite of $A n k^{\text {anklank }}$ mice also

48 contained markedly reduced levels of citrate and PPi and displayed diminished strength.

49 Together, our data show that ANKH is a crucial factor in extracellular citrate and PPi homeostasis

50 that is essential for normal bone development.

\section{Introduction}

Physiological mineralization is essential for normal development of vertebrates, but must

54 be restricted to specific sites of the body. Vertebrates have evolved mechanisms to allow 55 regulated mineralization in for instance bones and teeth, but prevent mineralization of soft 56 connective tissues ${ }^{1,2}$. The molecular details of the mechanism in vertebrates that restrict 57 mineralization to specific sites of the body are incompletely characterized, however.

The ANKHIAnk (human/mouse) gene encodes a multi-span transmembrane protein

59 involved in the prevention of pathological mineralization of cartilage and synovial fluid ${ }^{3,4}$.

60 ANKHIAnk, has a wide tissue distribution, which high levels of expression found in osteoblasts,

61 prostate, skeletal muscle, brain and the cardiovascular system ${ }^{1,5,6}$. A naturally occurring mouse

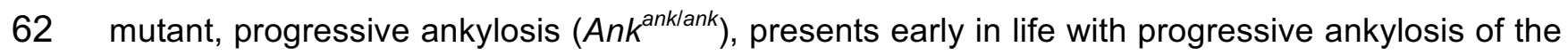

63 spine and other joints, restricting mobility and critically limiting lifespan ${ }^{1}$. Biallelic loss-of-function 
64 mutations in the human orthologue of Ank, Ank homolog (ANKH), underlie some forms of

65 craniometaphyseal dysplasia (CMD), which also presents with progressive ankylosis, mainly

66 affecting the spine and the joints of hands and feet ${ }^{7}$. In 2000 , Ho et al. showed that medium of

67 Ank $^{\text {anklank }}$ fibroblasts contained reduced concentrations of the physiological mineralization inhibitor

68 inorganic pyrophosphate (PPi), leading to the now prevailing view that ANKH/ANK was the

69 transport of PPi into the extracellular environment ${ }^{1,8}$. An important source of extracellular PPi is

70 ATP, which is extracellularly converted into AMP and PPi by membrane-bound ecto-nucleotidase

71 pyrophosphatase/phosphodiesterase 1 (ENPP1) ${ }^{9}$. We have previously shown that ATP release

72 mediated by the hepatic membrane protein ATP-Binding Cassette subfamily $\mathrm{C}$ member 6

73 (ABCC6) is responsible for 60-70\% of all PPi present in plasma ${ }^{10,11}$.

74 Here we tested if release of ATP also underlies most of the PPi found in the extracellular

75 milieu of ANKH-containing cells. Moreover, we applied global metabolite profiling ${ }^{12}$ on medium

76 of HEK293-ANKH cells to gain a comprehensive overview of metabolites extruded by cells in an

77 ANKH-dependent manner. Our results provide new and unexpected insights into the substrate

78 spectrum and anti-mineralization properties of $\mathrm{ANKH}$ and also show that $\mathrm{ANKH}$ has functions

79 beyond inhibition of inhibition of pathological mineralization as it is, for instance, essential for

80 normal bone development.

Results

83

84 HEK293-ANKH cells release ATP into the extracellular environment. To study the function of

85 ANKH in vitro, we first generated several HEK293 cell lines overproducing wild type ANKH

$86\left(\mathrm{ANKH}^{\mathrm{wt}}\right)$ and $\mathrm{ANKH} \mathrm{L}^{\mathrm{L} 24 \mathrm{~S}}$, a pathogenic loss-of-function mutant which still routes normally to the

87 plasma membrane ${ }^{7}$. As shown in Fig. $1 \mathrm{~A}$, endogenous $\mathrm{ANKH}$ was not detectable in parental

88 HEK293 cells by immunoblot analysis, whereas high levels of ANKH protein were found in cells

89 overexpressing $A N K H^{w t}$. The loss-of-function $A N K H^{L 244 S}$ mutant was also abundantly expressed, 
90 and a clone producing levels of the mutant protein higher than those detected in the HEK293-

$91 A N K H^{w t}$ cells was used for further analysis (Fig. 1A). First, we measured PPi levels in the medium

92 of these cells over a 24-h time period and showed that PPi accumulated at higher levels in medium

93 of HEK293-ANKH $H^{\text {wt }}$ cells than in medium of HEK293-ANKH ${ }^{L 244 S}$ or control HEK293 cells (Fig. 1B),

94 confirming earlier reports that demonstrated the involvement of ANKH in extracellular PPi

95 homeostasis ${ }^{1}$. We have previously shown that ENPP1 produced by HEK293 cells converts

96 extracellular ATP into AMP and $\mathrm{PPi}^{10}$. Consequently, to determine what part of the PPi found in

97 medium of $A N K H^{w t}$ cells might be derived from extracellular ATP, converted by ENPP1 into AMP

98 and PPi, AMP concentrations were quantified in the culture medium. As shown in Fig. 1C, a clear

99 time-dependent increase in AMP concentrations was detected in medium of HEK293-ANKH ${ }^{w t}$

100 cells, while medium of untransfected HEK293 parental cells or cells producing the loss-of-function

$101 A N K H^{L 244 S}$ mutant contained only very little AMP. PPi and AMP concentrations in medium of

$102 A N K H^{w t}$ cells were within the same range (1-2 $\mu \mathrm{M}$ after 12 hours, compare panels $\mathrm{B}$ and $\mathrm{C}$ of Fig.

103 1) and the ratio of PPi to AMP was very similar to that previously reported for HEK293 cells

104 overproducing ABCC6, a plasma membrane protein involved in the release of ATP ${ }^{10}$. We attribute

105 the somewhat lower abundance of AMP than PPi to further metabolism of AMP and the generation

106 of PPi from other nucleoside triphosphates (NTPs) also released into the culture medium via

107 ANKH (see below). A luciferase-based real-time ATP efflux assay was also carried out and

108 confirmed that ANKH is involved in cellular ATP release (Figure 1D). Only HEK293-ANKH ${ }^{\text {wt }}$ cells

109 showed robust ATP efflux, whereas release from HEK293-ANKH ${ }^{L 244 S}$ cells was indistinguishable

110 from untransfected parental HEK293 cells in these assays. Collectively, these data indicate that

111 HEK293-ANKH ${ }^{\text {wt }}$ cells release ATP, which is subsequently extracellularly converted into AMP and

112 PPi.

114 Culture medium of HEK293-ANKH $H^{\text {wt }}$ cells contains large amounts of nucleoside

115 monophosphates (NMPs). In addition to ATP, ENPP1 can convert various other nucleoside 
116 triphosphates (NTPs) into their respective nucleoside monophosphate (NMP) and PPi. Our

117 previous work has shown that ENPP1 activity in HEK293 cells is high ${ }^{10}$. We therefore used liquid 118 chromatography/mass spectrometry (LC/MS)-based global metabolite profiling to determine if 119 ANKH also provides a pathway for release of other NTPs. Substantially elevated levels of AMP, 120 CMP, GMP and UMP were detected in the culture medium of HEK293-ANKH ${ }^{\text {wt }}$ cells compared to 121 untransfected parental and HEK293-ANKH $H^{\text {L244S }}$ cells (Fig. 2 A-D), For AMP and UMP differences 122 between untranfected and HEK293-ANKH $H^{\text {wt }}$ cells reached statistical significance. These results 123 support the hypothesis that ANKH provides a previously unanticipated pathway for cellular NTP 124 release. Based on the levels of PPi, AMP and other NMPs detected in the culture medium, we estimate that cellular NTP release underlies at least $70 \%$ of the ANKH-dependent accumulation of PPi in the culture medium (for calculation see materials and methods section) of the PPi detected in medium of the HEK293-ANKH ${ }^{\text {wt }}$ cells.

HEK293-ANKH cells release the TCA cycle intermediates citrate, succinate, and malate into

the culture medium. The global metabolite profiling experiments also revealed that the calcium

131 chelator citrate specifically accumulated in the culture medium of HEK293-ANKH' cells (Fig. 3A).

132 Because global metabolite profiling experiments only provide relative metabolite levels, we also 133 quantified citrate levels by LC/MS in 24-hour medium samples and found that approximately 1 $134 \mathrm{mM}$ citrate $(2.5 \mu \mathrm{mol} / 24 \mathrm{hrs})$ was present in medium of HEK293-ANKH ${ }^{\text {wt }}$ cells, while it was almost 135 undetectable in medium of HEK293 control and HEK293-ANK ${ }^{\text {L244S }}$ cells. To put this in perspective, 136 the same medium samples of HEK293-ANKH ${ }^{\text {wt }}$ cells contained about $4 \mu \mathrm{M} \mathrm{PPi}$ (Fig. 1B), 137 equivalent to the release of approximately 10 nmoles of NTPs. Thus, the amount of citrate 138 released by the HEK293-ANKH ${ }^{w t}$ cells was at least 2 orders of magnitude higher than the amount 139 of NTPs. Other metabolites found to be selectively elevated in medium of HEK293-ANKH ${ }^{\text {wt }}$ cells 140 were malate (Fig. 3B) and succinate (Fig. 3C), although absolute levels increases relative to 141 control cells were clearly less than those found for citrate. Using an independent enzymatic assay, 
142 citrate levels in culture medium were also followed over time and as shown in Fig. 3D, these

143 experiments confirmed that citrate was present at approximately $1.1 \mathrm{mM}$ in the 24-hour culture

144 medium samples of the $A N K H^{w t}$ cells, comparable to the concentration determined by LC/MS.

145 Collectively these data show that ANKH is involved in the cellular release of large amounts of 146 citrate.

148 ANK affects PPi incorporation into bone

149 About $70 \%$ of the PPi found in plasma depends on ABCC6 activity ${ }^{11}$, indicating that the 150 contribution of ANKH/ANK to plasma PPi homeostasis is relatively minor. Consequently, instead 151 of contributing to central PPi homeostasis in plasma, we hypothesized that ANKH/ANK is 152 important in local PPi homeostasis. Osteoblasts express ANKH/ANK at relatively high levels ${ }^{5}$ and 153 the hydroxyapatite of bone contains substantial amounts of $\mathrm{PPi}^{13}$. To determine if ANK has a role 154 in incorporation of PPi in bone, we quantified PPi in tibiae and femora of wild type, Ank ${ }^{\text {anklank }}$, and 155 mice heterozygous for ank. As shown in Fig. 4 PPi constituted about $0.1 \%$ (weight/weight) of bone 156 tissue in wild type mice, whereas in Ank ${ }^{\text {anklank }}$ mice the amount of PPi associated with bone was 157 reduced by approximately $75 \%$. Moreover, in mice heterozygous for ank, PPi levels were also 158 moderately (by approximately $25 \%$ ), but significantly reduced. These data show that ANK is a 159 crucial factor in PPi homeostasis in the local environment of bone tissue.

161 ANKH affects citrate disposition in vivo. Plasma contains substantial amounts of citrate ${ }^{14}$. We 162 therefore determined the effect of a complete inactivation of ANK in mice on plasma citrate 163 concentrations and as shown in Fig. 5A, found that approximately $75 \%$ of citrate in plasma 164 depended on ANK. Because citrate is also one of the most abundant organic anions in urine ${ }^{15}$, 165 we measure citrate excretion in $A n k^{\text {anklank }}$ mice. As shown in Fig. 5B, the ank mutant mice excreted 166 approximately $40 \%$ less citrate via their urine than their wild type litter mates. The availability of 167 an NMR spectrum of urine of a 19-year-old female CMD patient carrying biallelic homozygous 
168 inactivating mutations in $A N K H\left(A N K H^{L 244 S}\right)$, previously described by Morava et al. ${ }^{7}$ made it 169 possible to carry out an analysis of citrate levels. Citric acid was not detected in urine of this CMD 170 patient (Fig. 5C, upper panel). The lower panel of Fig. 5C shows the typical citrate resonance in 171 urine of a representative age-matched control, which contained $370 \mu \mathrm{mol}$ citrate $/ \mathrm{mmol}$ creatinine.

172 It is interesting to note that the succinate resonance is visible in the NMR spectrum of control 173 urine, while its concentration is clearly much lower in urine of the CMD patient (Fig. 5E). These 174 data suggest that ANKH impacts the in vivo disposition of succinate and especially citrate in both, 175 humans and mice.

176 Like PPi, citrate is also one of the major organic compounds present in bone and also strongly 177 associates with hydroxyapatite ${ }^{16}$. With $90 \%$ of the body's citrate content present in bone, this 178 tissue is thought to play a central role in extracellular citrate homeostasis ${ }^{17}$. Therefore, we 179 determined if bone citrate levels depend on ANK. These experiments revealed that femora and 180 tibiae of $A n k^{\text {anklank }}$ mice contained approximately $50 \%$ less citrate than the same bones of wild 181 type mice (Fig. 5D,E). Moreover, bones of mice heterozygous for ank also contained less citrate, 182 which in the case of tibia was significantly lower than in wild type mice (Fig. 5D). Together these 183 data attest to the major impact of ANK on citrate homeostasis of bone.

185 Material properties of bone tissue of $A n k^{\text {anklank }}$ mice are altered. We next explored the 186 consequences of the absence of ANK activity on bone physiology, by characterizing geometry 187 and density of femurs harvested from Ank ${ }^{\text {anklank }}$, wild type and mice heterozygous for ank by 188 microCT. At 3 months of age, most of the bone parameters, including bone area (Fig. 6A), 189 tissue mineral density (TMD, Fig. 6B), and cortical thickness (Fig. 6C), were not significantly 190 different between wild type and $A n k^{\text {anklank }}$ mice. However, significant differences in cortical bone 191 properties between $A n k^{\text {anklank }}$ and wild type mice were detected for bone area fraction $(-12.1 \%)$, 192 cortical bone perimeter (+9.8\%), and cross-sectional geometry as indexed by eccentricity ($1939.4 \%)$. Next, the structural and material properties of the bone were determined by standard 
194 three-point bending. Plotting ultimate bending moment against section modulus (Fig. 6G)

195 yielded linear relationships for each genotype $\left(r^{2}=0.84\right.$ wild type, 0.73 HET, 0.67 Ank $\left.{ }^{\text {anklank }}\right)$ that

196 did not significantly differ in slope $(p=0.88)$. However, we observed that femurs from Ank ${ }^{\text {anklank }}$

197 mice required significantly less force per equivalent area of bone to break, as demonstrated by

198 a significant difference in regression intercept $(p=0.0170)$. Taken together, our results indicate

199 that the geometry of femora of $A n k^{\text {anklank }}$ mice is altered and that these femora have diminished

200 whole bone strength per equivalent amount of bone, results that are consistent with published

201 data showing citrate deposition in bone affects hydroxyapatite nanostructure and strength ${ }^{16}$.

Discussion

205 Ectopic mineralization - the deposition of hydroxyapatite in soft connective tissues - can be a

206 sequela of a number of clinical conditions, including aging, cancer, diabetes, chronic kidney

207 disease and genetic disorders. With no effective treatment currently available, ectopic

208 mineralization is associated with significant morbidity and mortality ${ }^{18}$.

209 ANKH/ANK is known for its important role in the prevention of pathological mineralization of joints,

210 and its absence results in severe, progressive, ankylosis in both, humans and mice. It was

211 previously thought that the main function of ANKH/ANK lies in regulation of extracellular PPi

212 homeostasis, but here we identified a new and previously unanticipated function of ANKH/ANK:

213 regulation of extracellular citrate concentrations. Although citrate has long been known to be a

214 major compound in plasma, urine and bone, the mechanism used by cells to extrude citrate has

215 been elusive. Our current data firmly link a specific protein, ANKH/ANK to extracellular citrate

216 disposition in vivo. Notably, our results are in line with previous GWAS studies describing a

217 correlation between plasma citrate levels and certain $A N K H$ variants in humans ${ }^{19}$ and an

218 association found in cows between intronic $A N K H$ variants and milk citrate concentrations ${ }^{20}$. 
219 Extracellular citrate is present in many tissues and body fluids where is serves diverse and, in 220 some cases, unknown functions ${ }^{14}$. In human plasma citrate levels are substantial $(100-300 \mu \mathrm{M})$,

221 but its function is unclear ${ }^{21}$. Various cell types express citrate uptake transporters and it has been

222 proposed that plasma citrate provides an additional energy source for cells under hypoglycemic

223 conditions ${ }^{14}$. Alternatively, citrate is a powerful anticoagulant and might prevent pathological

224 blood clotting.

225 Via glomerular filtration, plasma citrate ends up in urine, where it contributes to the prevention 226 kidney stone formation ${ }^{22}$. Whereas urine of the $A n k^{\text {ank/ank }}$ mice still contained substantial amounts 227 of citrate, that of the human CMD patient lacking functional ANKH was virtually devoid of citrate.

228 This difference might be partly explained by dietary differences: Citrate has a high bioavailability 229 of $80-90 \%{ }^{23}$ and is present in standard rodent food. Possibly, the human CMD patient had a diet 230 that was low in citrate, whereas part of the citrate detected in plasma of $A n k^{\text {ank/ank }}$ mice comes 231 from dietary sources.

232 Most of the body's citrate, over $90 \%$, is present in bone tissue, where it stabilizes hydroxyapatite

$233{ }^{16}$. Our results show that about $50 \%$ of bone citrate depends on ANK activity, in line with the 234 high expression of Ank in osteoblasts ${ }^{5}$. The altered material properties of Ank ${ }^{\text {ank/ank }}$ bones, i.e.

235 the altered relationship between ultimate moment and section modulus, nicely fits the described 236 role of citrate in stabilizing hydroxyapatite. The altered eccentricity and perimeter of Ank ${ }^{\text {anklank }}$

237 femora are most likely a result of compensatory bone remodeling to retain whole bone strength 238 without increasing total bone mass. Interestingly, Ma et al. recently reported that local levels of 239 extracellular citrate are also important for the osteogenic development of human mesenchymal 240 stem cells ${ }^{17}$. It is therefore conceivable that ANKH-dependent citrate release into bone is not 241 only important for the material properties of hydroxyapatite, but also contributes to osteogenic 242 differentiation.

243 Relatively high extracellular citrate concentrations of approximately $400 \mu \mathrm{M}$ are found in the 244 brain. Astrocytes actively release citrate, which is used by neurons as energy source under 
245 hypoglycemic conditions ${ }^{14}$. Patients suffering from CMD due to inactivating mutations in $A N K H$

246 suffer from mental retardation ${ }^{7}$, suggesting a function of ANKH in brain physiology.

247 The highest extracellular citrate concentrations are found in prostatic fluid (up to $180 \mathrm{mM}$ ).

248 ANKH is expressed at high levels in the epithelial cells of the prostate, known to release citrate

249 into prostatic fluid. Although a specific splice variant of the mitochondrial citrate carrier SLC25A1

250 has been implied in citrate efflux from the prostate ${ }^{24}$, ANKH likely contributes to this process.

251 In summary, extracellular citrate is present in many tissues and body fluids and we anticipate

252 that our discovery that ANKH/ANK is involved in extracellular citrate homeostasis will allow

253 clarifying its function in other tissues for instance by using the Ank ${ }^{\text {anklank }}$ mouse model.

255 A second important finding of the current study is that most, if not all, PPi found in the

256 extracellular environment of ANKH/ANK containing cells, originates from released NTPs, which

257 are extracellularly converted into their respective NMP and PPi by ENPP1. This contradicts

258 earlier work, proposing direct ANKH/ANK-dependent cellular efflux of $\mathrm{PPi}{ }^{1}$. Our current data

259 strongly support the conclusion that ANKH/ANK mediates release of NTPs release, not PPi.

260 First, in vitro experiments showed that the majority of PPi found in the culture medium of

261 HEK293-ANKH ${ }^{w t}$ cells was derived from NTP efflux. Two reports have appeared that suggest

262 cells release ATP in an ANKH-dependent manner ${ }^{25-27}$. These studies did however not quantify

263 the relative amounts of extracellular ATP, AMP and PPi and therefore did not allow assessment

264 of the relative contribution of ANKH-mediated ATP release to extracellular PPi concentrations.

265 Strong evidence arguing against direct PPi transport by ANKH/ANK also comes from our

266 analysis of bones of mice lacking ENPP1. Moreover, $75 \%$ of the PPi present in bone depends

267 on ANK activity (Fig. 4). If ANK would directly transport PPi, incorporation of this fraction into

268 bone would not require ENPP1 activity. However, we found that PPi is virtually absent in bones

269 of Enpp $1^{-/}$mice (asj GrsrJ) (Szeri et al, manuscript in preparation), which is only compatible with

270 ANKH/ANK mediating NTP release with subsequent extracellular formation of PPi by ENPP1. 
271 The function of PPi in bone tissue is not completely clear, but might be related to stabilization of

272 hydroxyapatite and, consequently, bone mineral density. Such a function would fit data of

273 previous studies showing that bones of Enpp $1^{-/}$mice, which virtually lack PPi (Szeri et al,

274 manuscript in preparation), have a substantially greater reduction in mineral density ${ }^{28,29}$ than

275 bones of $A n k^{\text {anklank }}$ mice. These data also indicate that the residual $25 \%$ of PPi found in bones of

276 Ank $k^{\text {anklank }}$ mice suffices to a large extent to keep BMD close to the normal range. The proposed

277 effects of PPi on mineral density are similar to the effects of bisphosphonates, pharmaceutical

$278 \mathrm{PPi}$ analogues that are widely used in the treatment of osteoporosis ${ }^{30}$. Kim et al ${ }^{5}$ have

279 previously found a more dramatic effect of ANK on bone mineral density. A different genetic

280 background of their $A n k^{\text {anklank }}$ mice might underlie this more dramatic effect. Food composition,

281 specifically varying PPi content ${ }^{31}$, might also have contributed to the differences found in BMD

282 between the two studies.

283

284 ANKH/ANK has previously been shown to inhibit ectopic mineralization in the microenvironment

285 of the joint space ${ }^{1}$. Low plasma levels of PPi underlie several other genetic mineralization

286 disorders ${ }^{18,32}$. Plasma citrate levels depend on ANK activity, demonstrating that ANK substrates

287 end up in the blood circulation. This does not come as a surprise given the wide tissue distribution

288 of ANKH/ANK ${ }^{1}$. Most likely, NTPs are also released into the blood circulation via ANK, allowing

289 subsequent PPi formation in plasma. Under normal conditions, $60-70 \%$ of plasma PPi comes

290 from ABCC6-mediated hepatic NTP secretion ${ }^{10,11}$. ANK can therefore be expected to be

291 responsible for part of the remaining $30-40 \%$ of the PPi present in plasma that is independent of

292 ABCC6 activity. The relatively small contribution of ANK together with the large variability in

293 plasma PPi concentrations ${ }^{10,11,31}$ prevents determination of the contribution of ANK to plasma PPi

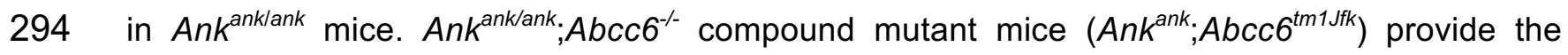

295 optimal experimental model system to determine the contribution of ANKH/ANK to plasma PPi. If

296 ANKH indeed contributes to plasma PPi homeostasis, it represents an attractive pharmacological 
297 target in ectopic mineralization disorders caused by low plasma levels of PPi. For instance, 298 stimulation of ANKH activity in patients suffering from pseudoxanthoma elasticum (PXE), a slowly 299 progressive ectopic calcification disorder caused by inactivating mutations in the gene encoding 300 the hepatic efflux transporter ABCC6 ${ }^{32}$, might increase plasma PPi concentrations and halt 301 disease progression. As citrate chelates calcium and has been shown to prevent kidney stone

302 (uroliths) formation ${ }^{22}$, ANKH-mediated citrate release might also contribute to inhibition of ectopic 303 mineralization in joints and other tissues. The previous observation of Ho et al. ${ }^{1}$ that Ank $k^{\text {anklank }}$ 304 mice have an increased incidence of kidney calcification would fit a function of ANKH/ANK in 305 prevention of ectopic mineralization in tissues different from those lining the joints.

307 In conclusion, we identified ANKH/ANK as an important player in cellular release of citrate and 308 NTPs. Citrate might have a previously unanticipated role in the prevention of soft tissue 309 mineralization, in addition to other major ectopic mineralization inhibitors like PPi, $\mathrm{Mg}^{2+}$ and 310 Fetuin-A ${ }^{33,34}$. Moreover, we found that ANKH/ANK is a crucial factor in normal bone physiology 311 by determining the amount of citrate and PPi incorporated in bone tissue.

\section{Materials and methods}

\section{Cell culture}

317 HEK293 cells were passaged in HyClone DMEM (GE) supplemented with 5\% FBS and 100 units 318 pen/strep per $\mathrm{ml}(\mathrm{Gibco})$ at $37^{\circ} \mathrm{C}$ and $5 \% \mathrm{CO}_{2}$ under humidified conditions. Efflux experiments 319 were performed in 6-well plates in $2.5 \mathrm{ml}$ Pro293a medium (Lonza) supplemented with 2 mM L320 glutamine and 100 units pen/strep per $\mathrm{ml}$.

\section{Animals}


323 Mice heterozygous for the progressive ankylosis allele (ank) were obtained from The Jackson

324 Laboratory (Bar Harbor, ME; C3FeB6 A/A $A^{w-J}-A n k^{a n k / J}$, stock number 000200). Heterozygote

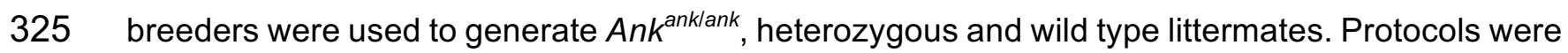

326 approved by the Institutional Animal Care and Use Committee of Thomas Jefferson University in

327 accordance with the National Institutes of Health Guide for Care and Use of Laboratory Animals.

328 Animals analyzed were between 11-14 weeks old. Plasma samples were collected by cardiac

329 puncture in heparinized syringes. Studies included similar numbers of male and female mice.

\section{Mutagenesis and overexpression of ANKH}

$332 A N K H^{w t}$ cDNA was obtained from Sino Biological and subcloned into pEntr223 by USER

333 cloning. The L244S mutation was introduced by USER cloning with primers 5'-

334 ACCAGAAGCuCAGCATCTTTCTTATTGTTGCATCTCCC-3' and

335 AGCTTCTGGUGGGCCTTCCGCTC TAATTCTGGCCACA. cDNAs were subsequently subcloned

336 in a Gateway compatible pQCXIP expression vector ${ }^{10}$. HEK293 cells were transfected with

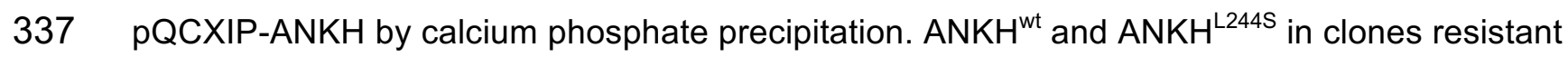

338 to $2 \mu \mathrm{M}$ puromycin were determined by immunoblot analysis, with a polyclonal antibody directed

339 against ANKH (OAAB06341, Aviva Systems Biology).

340

341 Enzymatic quantification of $\mathrm{PP}_{\mathrm{i}}, \mathrm{AMP}$ and citrate

342 In medium samples, $\mathrm{PP}_{\mathrm{i}}$ and $\mathrm{AMP}$ were quantified as described ${ }^{11}$ with modifications. PPi

343 concentrations were determined using ATP sulfurylase from NEB, and adenosine

344 5'phosphosulfate from Cayman Chemicals. AMP was quantified as follows: To $1 \mu$ l of sample or

345 standard, $100 \mu$ l of a solution containing $0.14 \mathrm{U} / \mathrm{ml}$ pyruvate orthophosphate dikinase (PPDK, kind

346 gift of Kikkoman Chemifa), $12.5 \mu \mathrm{mol} / \mathrm{L} \mathrm{PP}_{\mathrm{i}}$ (Sigma-Aldrich), $40 \mu \mathrm{mol} / \mathrm{L}$ phosphoenol pyruvate

347 (Cayman Chemicals), $50 \mu \mathrm{mol} / \mathrm{L}$ dithiothreitol, $1 \mathrm{mmol} / \mathrm{L}$ EDTA, $7.5 \mathrm{mmol} / \mathrm{L} \mathrm{MgSO}_{4}$ and $30 \mathrm{mmol} / \mathrm{L}$

348 BES ( $\mathrm{pH}$ 8.0) was added. Conversion of AMP into ATP was allowed to proceed for 20 min at 45 
$349{ }^{\circ} \mathrm{C}$, after which PPDK was inactivated by incubation at $80{ }^{\circ} \mathrm{C}$ for $10 \mathrm{~min}$. To determine PPi and 350 citrate amounts in bones, tibiae and femora of 13-week-old mice were collected and defleshed.

351 Epiphyses were removed and bone marrow was spun out of the bones $(30,000 \mathrm{RCF}, 1 \mathrm{~min})$.

352 Bones were subsequently dissolved by incubation with continuous mixing in $10 \%$ formic acid (60

$353{ }^{\circ} \mathrm{C}, 750 \mathrm{RPM}, 14 \mathrm{hrs}$ ). Samples were spun for $10 \mathrm{~min}$ at 30,000 RCF and the supernatant was

354 analyzed for PPi and citrate content. For bone extracts a slightly modified, more sensitive, version

355 of the PPi assay was used. A total reaction volume of $520 \mu$ l assay mix contained $100 \mu \mathrm{l}$ of SL-

356 ATP detection reagent (Biothema, Sweden), $0.1 \mu$ ATP removal reagent ("apyrase", BioThema,

357 Sweden), $6 \mu \mathrm{M}$ adenosine-5'-phosphosulphate (APS) (SantaCruz, TX), 0.15 U/ml ATP

358 sulphurylase (ATPS) (New England Biolabs) and $400 \mu$ l of ATP-free Tris-EDTA buffer (BioThema,

359 Sweden) was first incubated overnight at room temperature to convert PPi into ATP for 360 subsequent degradation by apyrase. The overnight incubation removed background PPi from the 361 assay mixture, resulting in a higher sensitivity of the assay. Next, the sample, diluted 500 -fold in 362 Tris-EDTA buffer, was added to $500 \mu \mathrm{l}$ of the assay mixture, resulting in an increase in 363 luminescence due to the conversion of PPi and APS into ATP, a reaction catalyzed by ATPS.

364 Finally, a known amount of ATP was added as internal standard and the ratio between the

365 increase in bioluminescent signal induced by the addition of PPi and by the increase induced by

366 the addition of ATP was used to calculate the PPi concentration. The assay was performed in a

367 Berthold FB12 luminometer in the linear range of the detector. Internal PPi standards were used

368 to show robustness and sensitivity of the assay.

369 Citrate was quantified in medium samples using the Megazyme Citric Acid Kit (Megazyme, 370 Ireland).

\section{Real-time ATP efflux assay}

373 Real-time ATP efflux assays were performed as described ${ }^{11}$, with modifications. To reduce ATP 374 release by the initial buffer change, cells were incubated at $27^{\circ} \mathrm{C}$, for $1 \mathrm{hr}$. Then an additional 50 
$375 \mu$ of ATP efflux buffer containing $10 \%$ of ATP-monitoring reagent (BactiterGlo, Promega),

376 dissolved in ATP efflux buffer was added. Bioluminescence was followed in real-time for $1 \mathrm{hr}$ at

$37727^{\circ} \mathrm{C}$ and $2 \mathrm{hrs}$ at $37^{\circ} \mathrm{C}$ in a Flex Station3 microplate reader (Molecular Devices).

379 LC/MS-based global metabolite profiling

380 Proteins were precipitated in $200 \mu \mathrm{l}$ of medium or $50 \mu \mathrm{l}$ plasma by adding $800 \mu \mathrm{l}$ and $200 \mu \mathrm{l}$

381 acetonitrile:methanol (1:1), respectively. Samples were shaken (10 minutes, $\left.500 \mathrm{RPM}, 21^{\circ} \mathrm{C}\right)$,

382 centrifuged $\left(15,000 \mathrm{~g}, 4^{\circ} \mathrm{C}, 10 \mathrm{~min}\right)$ and the supernatant dried in a Speed-Vac. Pellets were

383 stored at $-20^{\circ} \mathrm{C}$ until analysis. For analysis pellets were suspended in $45 \mu$ mobile phase $\mathrm{A}$ of

384 which $10 \mu \mathrm{l}$ was analyzed by ion-pairing LC/MS as described ${ }^{12}$.

385 Analytes were identified based on accurate mass and retention time, which matched reference

386 standards. Peak areas were determined using Masshunter Qualitative Analysis software version

387 7.0SP2 (Agilent Technologies).

388

\section{LC/MS-based quantification of citrate}

390 Plasma proteins were removed as described above and resuspended in 50 ul mobile phase A, 391 while urine and bone samples were diluted in mobile phase A (5 and 20-fold, respectively). A 392 volume of $5 \mu \mathrm{l}$ of each sample was analyzed as described under LC/MS global metabolite 393 profiling, along with calibration curves consisting of mobile phase A spiked with citrate 394 concentrations ranging from 1 to $1000 \mu \mathrm{M}$. Quantification was performed using Masshunter 395 Profinder Quantitative Analysis software version B.08.00, service pack 3 (Agilent Technologies).

\section{$397 \quad$ NMR spectroscopy}

398 One-dimensional ${ }^{1} \mathrm{H}-\mathrm{NMR}$ spectroscopy of urine samples was performed as described ${ }^{35}$. Briefly, 399 urine samples were centrifuged for $10 \mathrm{~min}$ at $3,000 \mathrm{~g}$ and trimethylsilyl-2,2,3,3400 tetradeuteropropionic acid (TSP; sodium salt; Sigma) in $\mathrm{D}_{2} \mathrm{O}$ was added before analysis to serve 
401 both, as an internal quantity reference and a chemical shift reference. The $\mathrm{pH}$ of each sample

402 was adjusted to $2.50 \pm 0.05$ with concentrated $\mathrm{HCl} .{ }^{1} \mathrm{H}-\mathrm{NMR}$ spectra were obtained using a Bruker

403 500-MHz spectrometer (pulse angle: $90^{\circ}$; delay time: 4 s; no. of scans: 256 ; relaxation delay: 2s).

404 Assignment of peak positions for compound identification was performed by comparing the peak

405 positions in the spectra of the metabolites with the reference spectral database of model

406 compounds at pH 2.5 using Amix version 3.9.14 (Bruker BioSpin).

407

408 Calculation of the contribution of NTP release to ANKH-dependent accumulation of PPi in 409 the culture medium

410 To estimate the contribution of $\mathrm{ANKH}^{\mathrm{wt}}$-mediated NTP release to 24-hour extracellular PPi 411 concentrations, PPi concentrations in medium of HEK293 parental cells were subtracted from the $412 \mathrm{PPi}$ concentrations detected in medium of HEK293-ANKH ${ }^{\text {wt }}$ cells, yielding an ANKH-specific PPi 413 accumulation in the $24-\mathrm{hr}$ culture medium samples of $2.4 \mu \mathrm{M}$. The same calculation demonstrated 414 an ANKH-specific accumulation of $1.4 \mu \mathrm{M}$ AMP in the culture medium. This demonstrated that 415 ATP release underlies at least $60 \%$ of the ANKH-dependent PPi accumulation detected in the 416 culture medium $(1.4 / 2.4 \times 100=58)$. GMP, UMP and CMP were also found to increase in culture 417 medium in an ANKH-dependent manner. Based on the relative LC/MS signals of the NMPs, we 418 estimated that AMP was responsible for $80 \%$ of the total NMP concentration in the culture 419 medium, whereas GMP, UMP and CMP together were responsible for the remaining $20 \%$. 420 Together these data demonstrate that nucleoside monophosphate (NMP) concentrations could 421 explain $70 \%$ of the ANKH-dependent PPi that had accumulated in the culture medium after 24 422 hrs. The calculated $70 \%$ is most likely an underestimation, as generated NMPs will be further 423 metabolized by the HEK293 cells, as we have observed before ${ }^{10}$.

\section{MicroCT}


426 Each bone was scanned using a Bruker Skyscan 1275 microCT system equipped with a $1 \mathrm{~mm}$

427 aluminum filter. One femur from each mouse was scanned at $55 \mathrm{kV}$ and $181 \mu \mathrm{A}$ with a $74 \mathrm{~ms}$

428 exposure time. Transverse scan slices were obtained by placing the long axis of the bone

429 parallel to the $z$ axis of the scanner using a custom 3D printed sample holder. An isometric

430 voxel size of $13 \mu \mathrm{m}$ was used. Images were reconstructed using nRecon (Bruker) and analyzed

431 using CTan (Bruker).

\section{Three-point Bending assay}

434 Three-point bending was performed on bones that had been stored at $-20^{\circ} \mathrm{C}$ in PBS-soaked

435 gauze after harvest. Femora were scanned with microCT before performing three-point bending.

436 Briefly, each femur was oriented on a standard fixture with femoral condyles facing down and a

437 bending span of $8.7 \mathrm{~mm}$. Next, a monotonic displacement ramp of $0.1 \mathrm{~mm} / \mathrm{s}$ was applied until

438 failure, with force and displacement acquired digitally. The force-displacement curves were

439 converted to stress-strain using microCT-based geometry and analyzed using a custom GNU

440 Octave script.

441

442 Statistical analyses.

443 P-values of group comparisons were calculated using one-way Anova using Prism 7.0d version

444 (GraphPad Software Inc.). Significance is indicated in the figures, with ${ }^{*}<0.05,{ }^{* *}<0.01,{ }^{* * *}<$

$445 \quad 0.001$ and $^{* * * *}<0.0001$.

446

448 Author contributions

449 FS Wrote manuscript, performed experiments

450 SL Performed experiments

451 SD Performed experiments 
452 UE Performed NMR analysis of urine samples

453 RE Provided access to essential equipment

454 KR Provided access to critical equipment

$455 \mathrm{CW} \quad$ Provided essential reagents

456 JPS Provided essential reagents

457 RW Provided patient sample and analyzed data

458 RET performed experiments, analyzed data

459 RJ Performed metabolomics analyses, analyzed data

460 KvdW Wrote manuscript, analyzed data, conceptualized project, supervised project

462 All Authors have seen and reviewed the manuscript

463

\section{Acknowledgements}

465 We thank our colleagues Piet Borst (The Netherlands Cancer Institute), Susan Cole (Queens's

466 University) and Jouni Uitto (Thomas Jefferson University) for critically reviewing our manuscript

467 and valuable discussions. FSz received financial support from the Fulbright Visiting Scholar

468 Program sponsored by the U.S. Department of State and a mobility grant from the Hungarian

469 Academy of Sciences. Further funding for this work was provided by PXE International and the

470 National Institutes of Health Grant R01AR072695 (KvdW).

471

472 Competing Interests

473 The authors declare they have no financial or non-financial competing interests. 


\section{References}

1. Ho, A. M., Johnson, M. D. \& Kingsley, D. M. Role of the mouse ank gene in control of tissue calcification and arthritis. Science 289, 265-270 (2000).

2. Kawasaki, K., Buchanan, A. V. \& Weiss, K. M. Biomineralization in humans: making the hard choices in life. Annu. Rev. Genet. 43, 119-142 (2009).

3. Gurley, K. A. et al. Mineral formation in joints caused by complete or joint-specific loss of ANK function. J. Bone Miner. Res. 21, 1238-1247 (2006).

4. Abhishek, A. \& Doherty, M. Pathophysiology of articular chondrocalcinosis--role of ANKH. Nat. Rev. Rheumatol. 7, 96-104 (2011).

5. Kim, H. J., Minashima, T., McCarthy, E. F., Winkles, J. A. \& Kirsch, T. Progressive ankylosis protein (ANK) in osteoblasts and osteoclasts controls bone formation and bone remodeling. J. Bone Miner. Res. 25, 1771-1783 (2010).

6. Wu, C. et al. BioGPS: an extensible and customizable portal for querying and organizing gene annotation resources. Genome Biol. 10, R130-8 (2009).

7. Morava, E. et al. Autosomal recessive mental retardation, deafness, ankylosis, and mild hypophosphatemia associated with a novel ANKH mutation in a consanguineous family. J. Clin. Endocrinol. Metab. 96, E189-98 (2011).

8. Orriss, I. R., Arnett, T. R. \& Russell, R. G. G. Pyrophosphate: a key inhibitor of mineralisation. Curr. Opin. Pharmacol. 28, 57-68 (2016).

9. Nitschke, Y. \& Rutsch, F. Inherited Arterial Calcification Syndromes: Etiologies and Treatment Concepts. Curr. Osteoporos. Rep. 15, 1-16 (2017).

10. Jansen, R. S. et al. ABCC6 prevents ectopic mineralization seen in pseudoxanthoma elasticum by inducing cellular nucleotide release. Proc. Natl. Acad. Sci. U.S.A. 110, 20206-20211 (2013).

11. Jansen, R. S. et al. ABCC6-mediated ATP secretion by the liver is the main source of the mineralization inhibitor inorganic pyrophosphate in the systemic circulation-brief report. Arterioscler. Thromb. Vasc. Biol. 34, 1985-1989 (2014).

12. Goncalves, M. D. et al. High-fructose corn syrup enhances intestinal tumor growth in mice. Science 363, 1345-1349 (2019).

13. Alfrey, A. C. \& Solomons, C. C. Bone pyrophosphate in uremia and its association with extraosseous calcification. J. Clin. Invest. 57, 700-705 (1976).

14. Mycielska, M. E., Milenkovic, V. M., Wetzel, C. H., Rümmele, P. \& Geissler, E. K. Extracellular Citrate in Health and Disease. Curr. Mol. Med. 15, 884-891 (2015).

15. Petrarulo, M., Facchini, P., Cerelli, E., Marangella, M. \& Linari, F. Citrate in urine determined with a new citrate lyase method. Clin. Chem. 41, 1518-1521 (1995).

16. Hu, Y.-Y., Rawal, A. \& Schmidt-Rohr, K. Strongly bound citrate stabilizes the apatite nanocrystals in bone. Proc. Natl. Acad. Sci. U.S.A. 107, 22425-22429 (2010).

17. Ma, C. et al. Citrate-based materials fuel human stem cells by metabonegenic regulation. Proc. Natt. Acad. Sci. U.S.A. 115, E11741-E11750 (2018).

18. Uitto, J., Li, Q., van de Wetering, K., Váradi, A. \& Terry, S. F. Insights into Pathomechanisms and Treatment Development in Heritable Ectopic Mineralization Disorders: Summary of the PXE International Biennial Research Symposium-2016. J. Invest. Dermatol. 137, 790-795 (2017).

19. Shin, S.-Y. et al. An atlas of genetic influences on human blood metabolites. Nat. Genet. 46, 543-550 (2014).

20. Sanchez, M.-P. et al. Sequence-based GWAS, network and pathway analyses reveal genes co-associated with milk cheese-making properties and milk composition in Montbéliarde cows. Genet. Sel. Evol. 51, 34-19 (2019).

21. Rudman, D. et al. Hypocitraturia in patients with gastrointestinal malabsorption. N. Engl. J. Med. 303, 657-661 (1980).

22. Raffin, E. P. et al. The Effect of Thiazide and Potassium Citrate Use on the Health 
23. Harvey, J. A., Zobitz, M. M. \& Pak, C. Y. Bioavailability of citrate from two different preparations of potassium citrate. J. Clin. Pharmacol. 29, 338-341 (1989).

24. Mazurek, M. P. et al. Molecular origin of plasma membrane citrate transporter in human prostate epithelial cells. EMBO reports 11, 431-437 (2010).

25. Rosenthal, A. K. et al. The progressive ankylosis gene product ANK regulates extracellular ATP levels in primary articular chondrocytes. Arthritis Res. Ther. 15, R154 (2013).

26. Costello, J. C. et al. Parallel regulation of extracellular ATP and inorganic pyrophosphate: roles of growth factors, transduction modulators, and ANK. Connect. Tissue Res. 52, 139-146 (2011).

27. Mitton-Fitzgerald, E., Gohr, C. M., Bettendorf, B. \& Rosenthal, A. K. The Role of ANK in Calcium Pyrophosphate Deposition Disease. Curr. Rheumatol. Rep. 18, 25 (2016).

28. Albright, R. A. et al. ENPP1-Fc prevents mortality and vascular calcifications in rodent model of generalized arterial calcification of infancy. Nature Commun. 6, 10006 (2015).

29. Li, Q. et al. Mutant Enpp1asj mice as a model for generalized arterial calcification of infancy. Dis. Models Mech. 6, 1227-1235 (2013).

30. Russell, R. G. G. Bisphosphonates: From Bench to Bedside. Ann. N. Y. Acad. Sci. 1068, 367-401 (2006).

31. Dedinszki, D. et al. Oral administration of pyrophosphate inhibits connective tissue calcification. EMBO Mol. Med. 9, 1463-1470 (2017).

32. Borst, P., Váradi, A. \& van de Wetering, K. PXE, a Mysterious Inborn Error Clarified. Trends Biochem. Sci. 44, 125-140 (2019).

33. Herrmann, M., Kinkeldey, A. \& Jahnen-Dechent, W. Fetuin-A Function in Systemic Mineral Metabolism. TCM 22, 197-201 (2012).

34. Rüfenacht, H. S. \& Fleisch, H. Measurement of inhibitors of calcium phosphate precipitation in plasma ultrafiltrate. Am. J. Physiol. 246, F648-55 (1984).

35. Engelke, U. F. H. et al. Guanidinoacetate methyltransferase (GAMT) deficiency diagnosed by proton NMR spectroscopy of body fluids. NMR Biomed. 22, 538-544 (2009).

36. Kirejczyk, J. K. et al. Urinary citrate excretion in healthy children depends on age and gender. Pediatr. Nephrol. 29, 1575-1582 (2014). 
560 Fig 1. HEK293-ANKH $H^{w t}$ cells release ATP, which is rapidly converted into pyrophosphate

561 (PPi) and AMP. Detection of ANKH in HEK293 parental, HEK293-ANKH ${ }^{w t}$ and HEK293$562 A N K H^{L 244 S}$ cells by immunoblot analysis (A). Concentrations of pyrophosphate (PPi) (B) and AMP

563 (C) were quantified enzymatically and followed in medium samples of HEK293 parental, HEK293-

$564 \mathrm{ANKH}^{\mathrm{wt}}$ and HEK293-ANKH ${ }^{\mathrm{L} 244 S}$ cells over the course of 24 hours. ATP release by HEK293

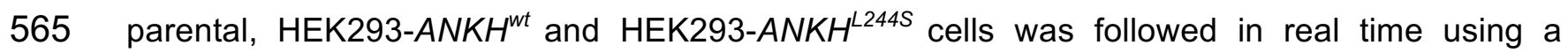

566 luciferase-based assay (D). Results of representative experiments performed in triplicate are

567 shown. In panels B and C data are expressed as mean +/- SD. Panel D shows mean +/- SEM.

568

569 Fig 2. Medium of HEK293-ANKH ${ }^{\text {wt }}$ cells contains large amounts of nucleoside

570 monophosphates. LC/MS-based global metabolite profiling was applied to 24-hour medium

571 samples of HEK293 parental, HEK293-ANKH $H^{w t}$ and HEK293-ANKH ${ }^{L 244 S}$ cells. The relative

572 abundance of masses corresponding to AMP (A), CMP (B), GMP (C) and UMP (D) were

573 determined. Authentic standards were used to confirm the identity of NMPs. Data are expressed

574 as mean $+/-$ SD of an experiment performed in triplicate. ${ }^{*} p<0.05,{ }^{* *} p<0.01$.

575

576 Fig 3. Medium of HEK293-ANKH ${ }^{w t}$ cells contains large amounts of citrate, succinate and

577 malate. LC/MS-based global metabolite profiling was applied to 24-hour medium samples of 578 HEK293 parental, HEK293-ANKH ${ }^{w t}$ and HEK293-ANKH $H^{L 244 S}$ cells. The relative abundance of 579 masses corresponding to citrate (A), malate (B) and succinate (C) were determined. Authentic 580 standards were used to confirm the identity of the Krebs-cycle intermediates. Using an

581 enzymatic assay, citrate concentrations were followed for 24 hours in (D). Data are expressed

582 as mean $+/$ - SD of an experiment performed in triplicate. ${ }^{* * *} p<0.0001$. 
584 Fig 4. PPi content of bone tissue depends on ANK activity. Pyrophosphate content of tibiae

585 (A) and femora (B) of wild type $(\mathrm{n}=10)$, heterozygous $(\mathrm{HET}, \mathrm{n}=10)$ and Ank $^{\text {anklank }}$ (ank/ank, $\left.\mathrm{n}=8\right)$

586 mice. Data are expressed as mean $+/-$ SD. ${ }^{* *} p<0.01,{ }^{* * *} p<0.0001$.

588 Fig 5. Extracellular Citrate depends on ANKIANKH activity. (A) Citrate plasma concentrations 589 in wild type $(n=8)$, heterozygous $(\mathrm{HET}, \mathrm{n}=8)$ and $A n k^{\text {anklank }}(\mathrm{n}=8)$ mice. Citrate concentrations in 590 urine of wild type $(n=6)$, heterozygous $(H E T, n=10)$ and $A n k^{\text {anklank }}(n=9)$ mice. (C) Urine of a patient 591 suffering from craniometaphyseal dysplasia (CMD) due to biallelic inactivating mutations in $A N K H$ 592 is virtually devoid of citrate. NMR spectra of urine of a patient with biallelic 593 pathogenic $A N K H^{\mathrm{L244S}}$ mutations (C, upper panel). A representative sex- and age-matched control 594 urine sample contained $370 \mu \mathrm{mol} / \mathrm{mmol}$ creatinine (C, lower panel). Spectra are scaled on 595 creatinine. Citrate resonates as a typical AB-system (2.98 ppm; four peaks between 2.80 and

$5963.05 \mathrm{ppm})$. Reference values for urinary citrate for this age group are $208-468 \mu \mathrm{mol} / \mathrm{mmol}$ 597 creatinine ( $\mathrm{n}=20$ healthy controls) ${ }^{36}$. Succinate resonates as a singlet resonance at $2.66 \mathrm{ppm}$.

598 For unknown reasons, urinary lactate was somewhat increased in urine of the CMD patient $599(120 \mu \mathrm{mol} / \mathrm{mmol}$ creatinine; reference $<75 \mu \mathrm{mol} / \mathrm{mmol}$ creatinine $)$. Citrate content of tibiae (D) and 600 femora $(E)$ of wild type $(n=10)$, heterozygous (HET, $n=10)$ and Ank ${ }^{\text {anklank }}(n=10)$ mice. Data are 601 expressed as mean $+/-$ SD. ${ }^{*} p<0.05,{ }^{* *} p<0.01,{ }^{* * *} p<0.001,{ }^{* * * *} p<0.0001$.

602

603 Fig 6. Bone geometry and mechanical performance is altered in the absence of ANK 604 activity. microCT was used to determine (A) bone area, $(B)$ cortical thickness, $(C)$ tissue mineral 605 density (TMD), (D) bone area fraction (B.Ar/T.Ar), (E) bone perimeter, and (F) eccentricity in 606 femora of wild type $(n=9)$, heterozygous (HET, $n=10)$ and Ank ${ }^{\text {anklank }}(n=8)$ mice. $(G)$ To compare 607 whole bone bending strength, a linear regression between section modulus and ultimate bending 608 moment was analyzed for each genotype $\left(r^{2}=0.84\right.$ wild type, 0.73 HET, 0.67 Ank $\left.{ }^{\text {anklank }}\right)$. The 609 slope was not different between genotypes, but the intercept was significantly different in femora 
bioRxiv preprint doi: https://doi.org/10.1101/2019.12.20.883223; this version posted December 20, 2019. The copyright holder for this preprint (which was not certified by peer review) is the author/funder. All rights reserved. No reuse allowed without permission.

610 from $A n k^{\text {anklank }}$ mice, which utilized an increased section modulus to achieve the corresponding

611 ultimate moment. ${ }^{*} p<0.05,{ }^{* *} p<0.01,{ }^{* * *} p<0.001$.

612 


\section{Figure 1}

A
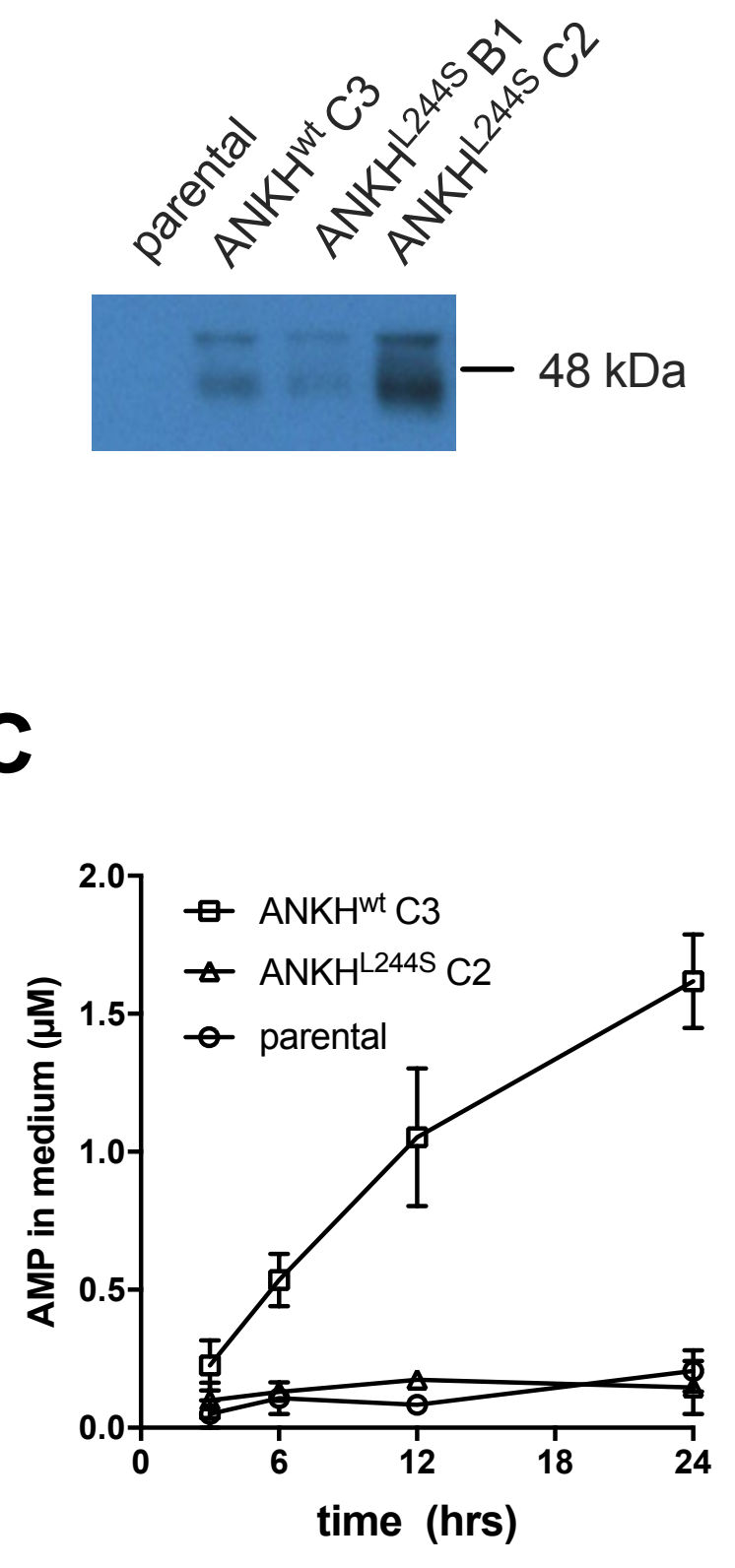

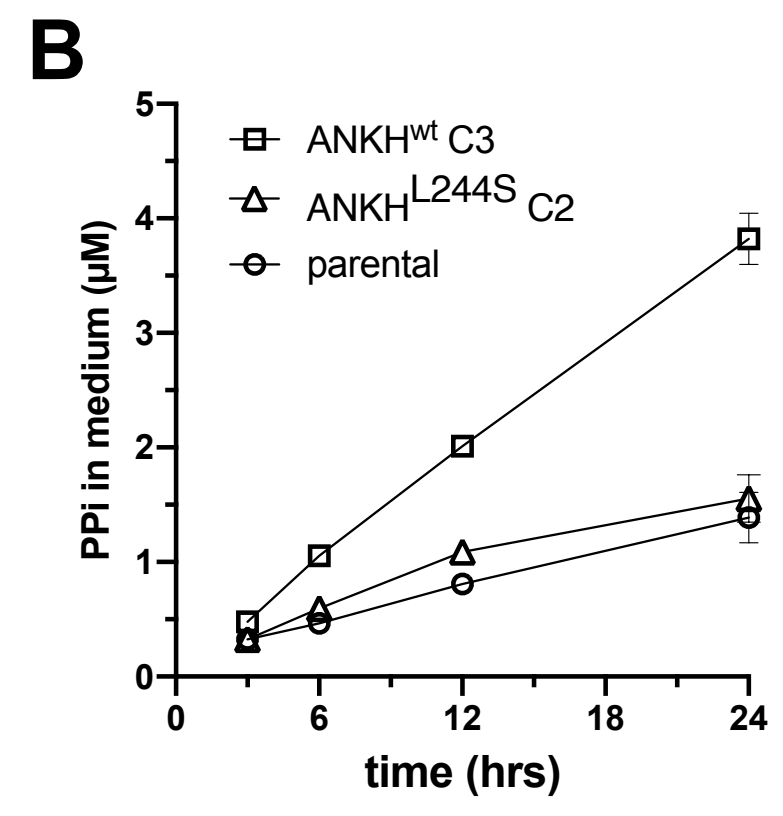

D

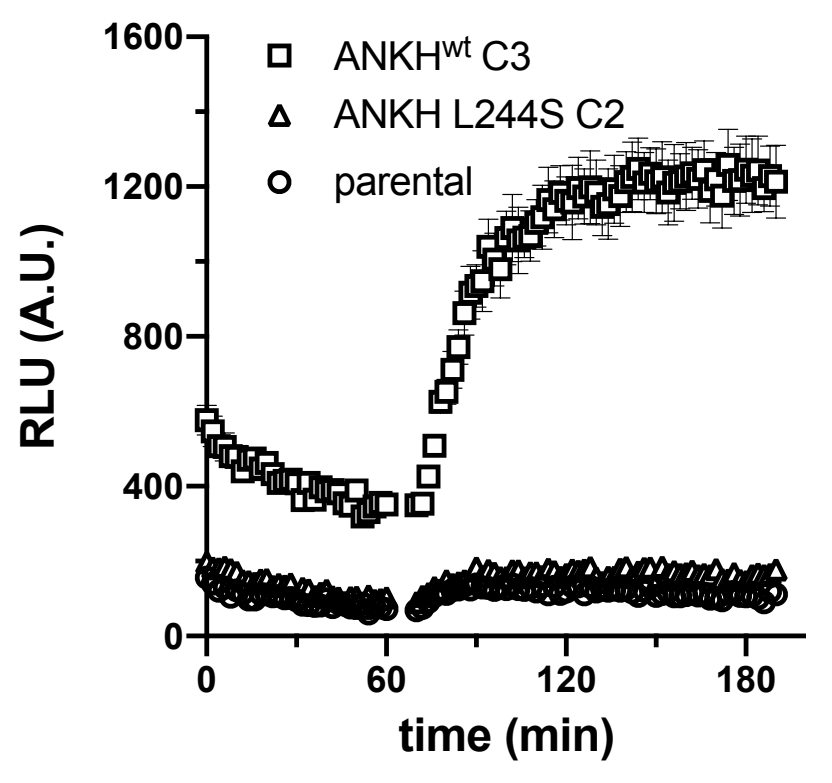




\section{Figure 2}

A

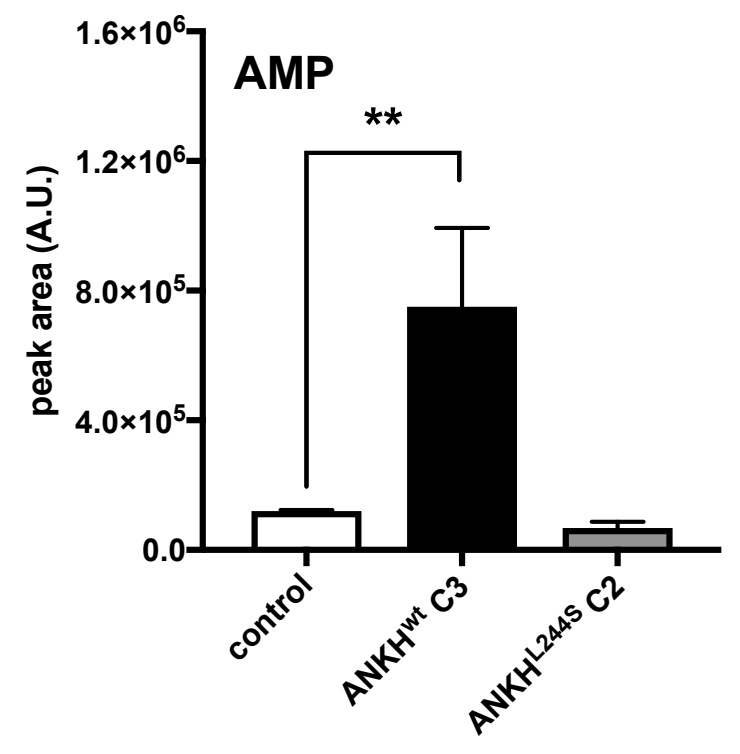

C

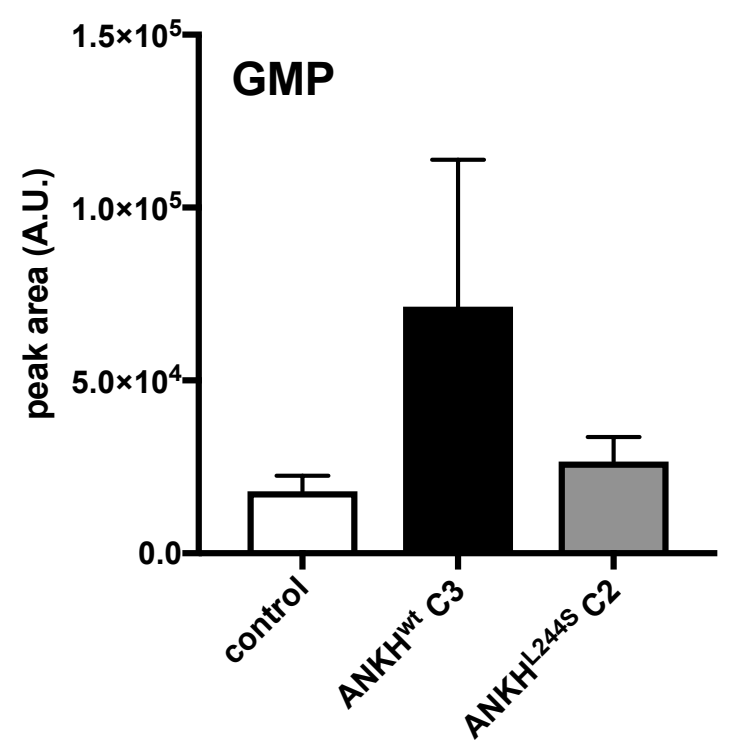

B

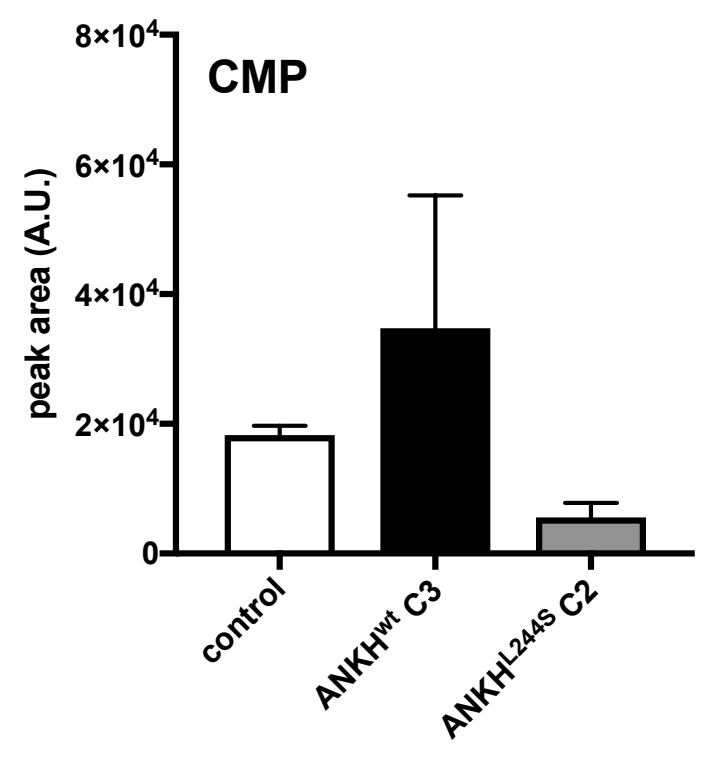

D

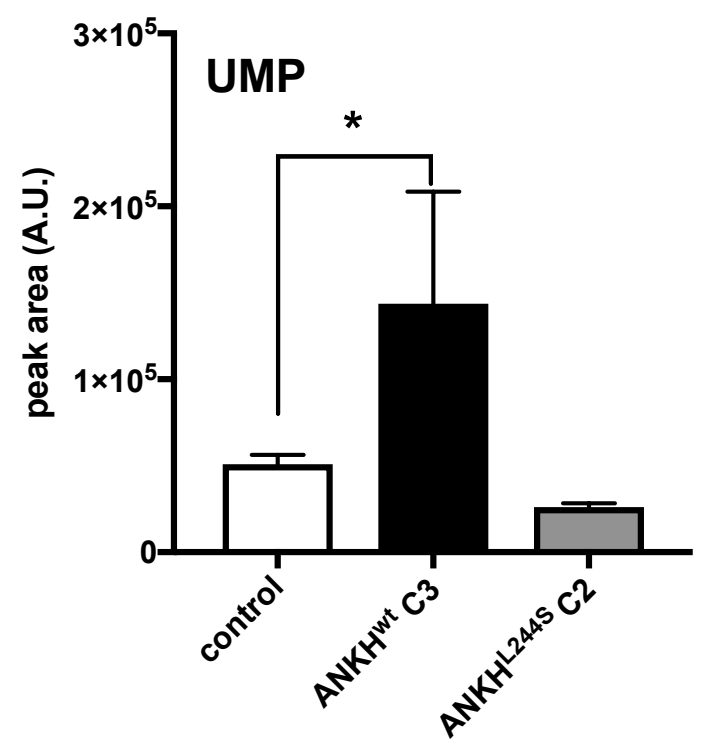




\section{Figure 3}

A

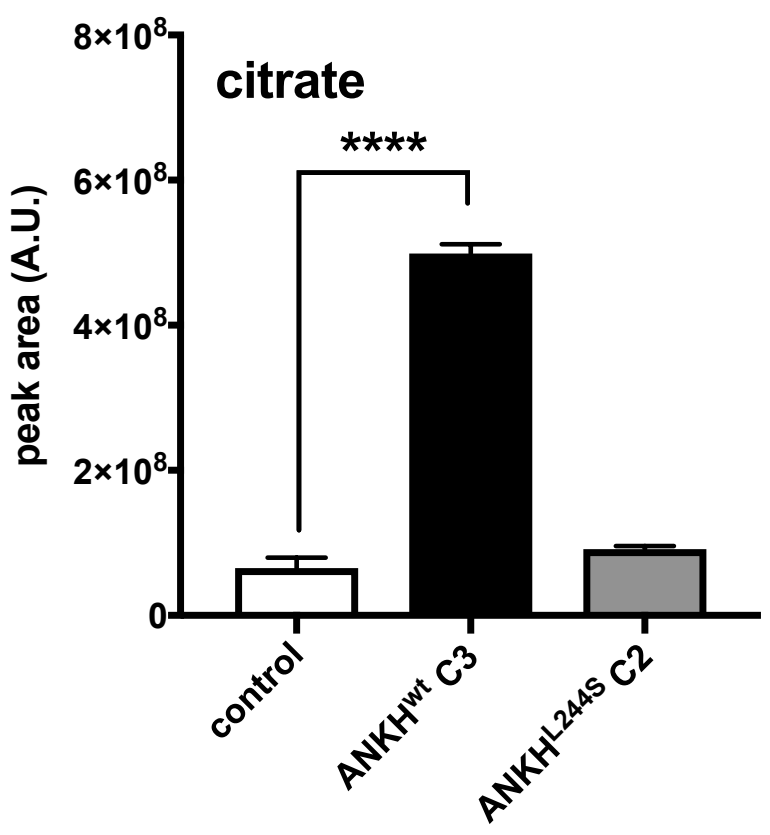

C

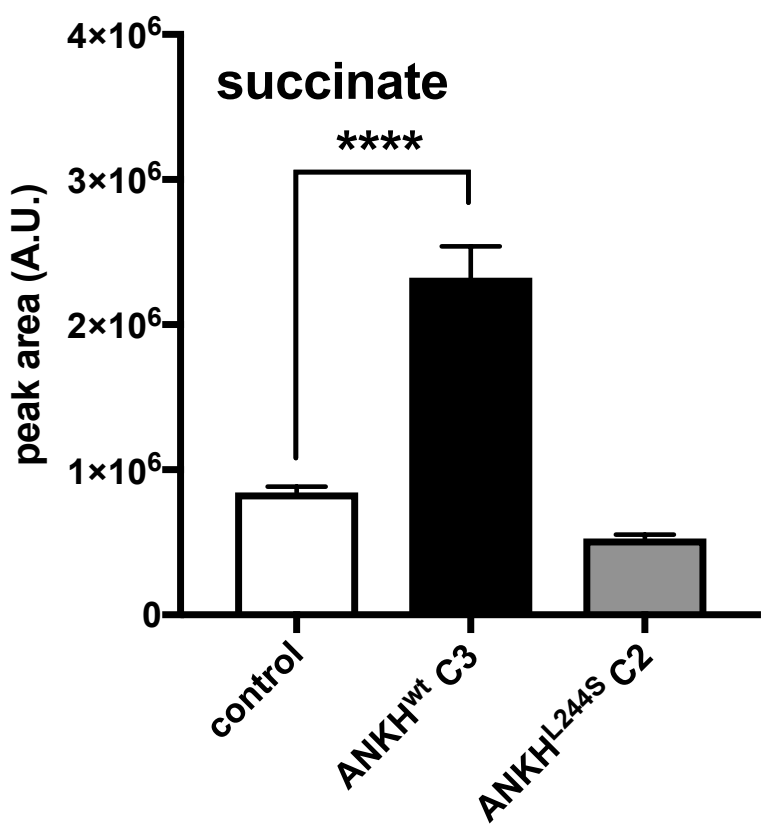

B

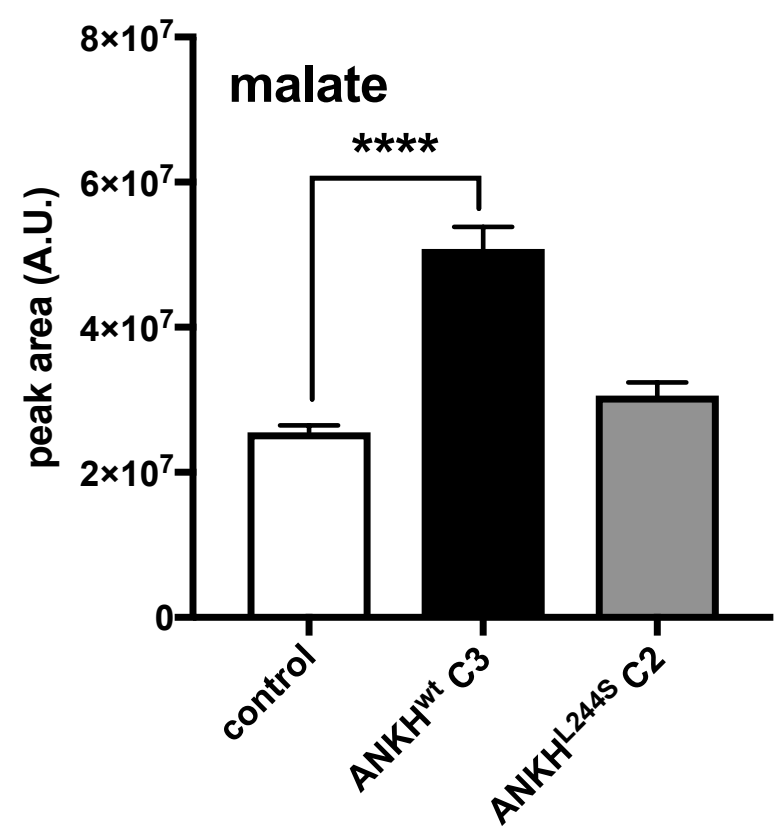

D

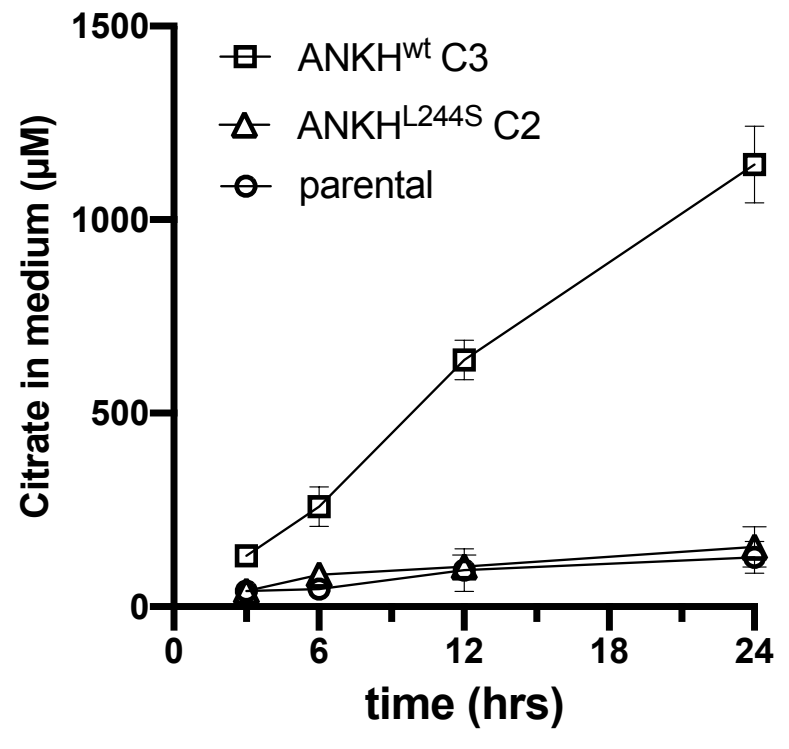




\section{Figure 4}

A

tibia

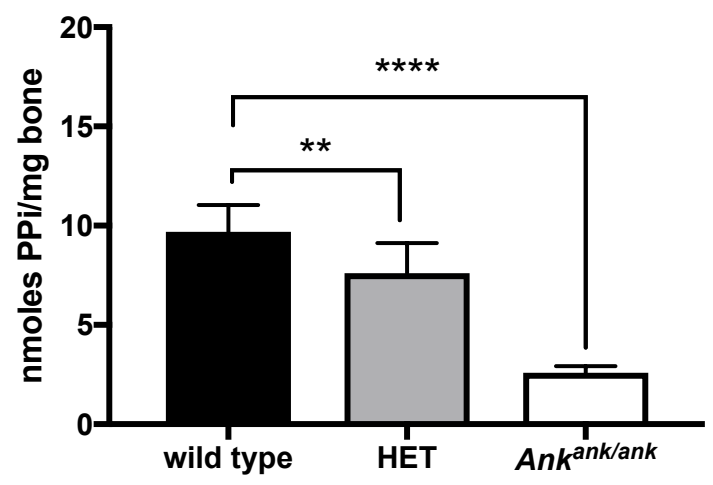

B
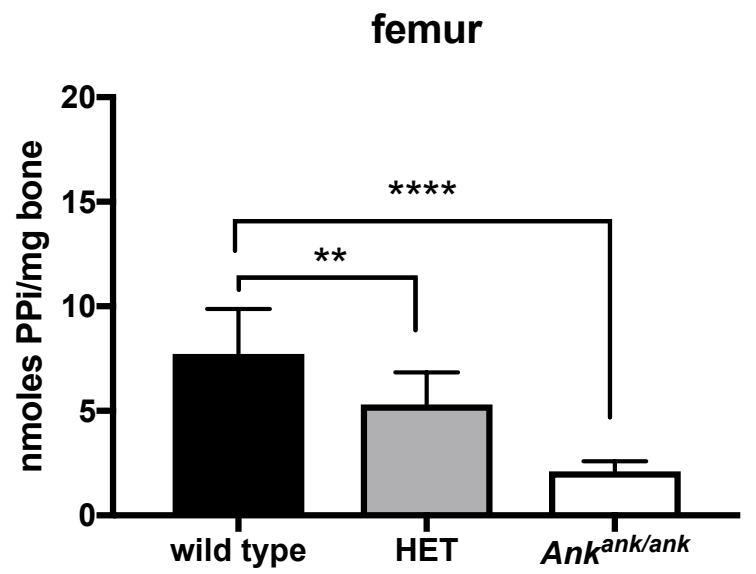

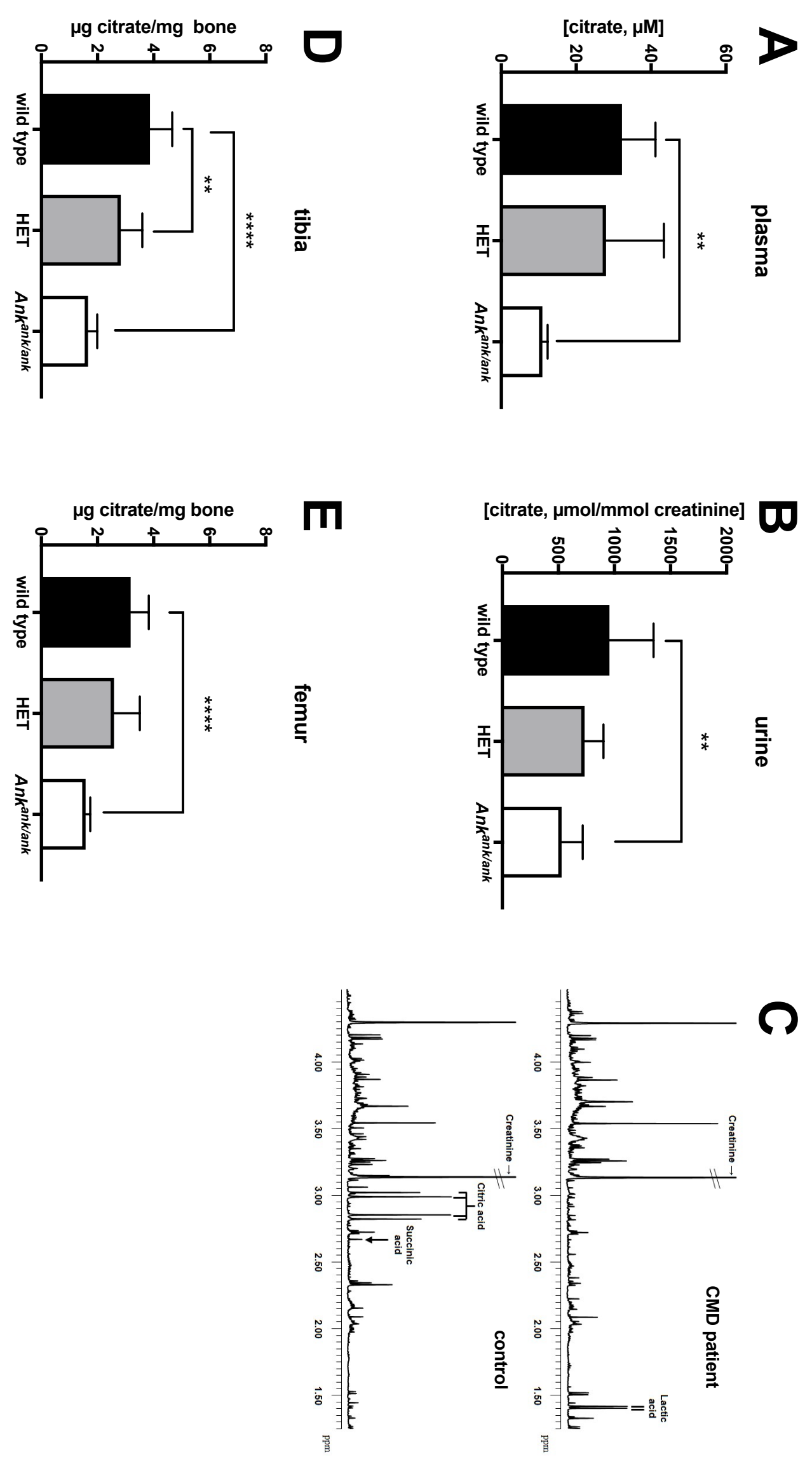


\section{Figure 6}

A

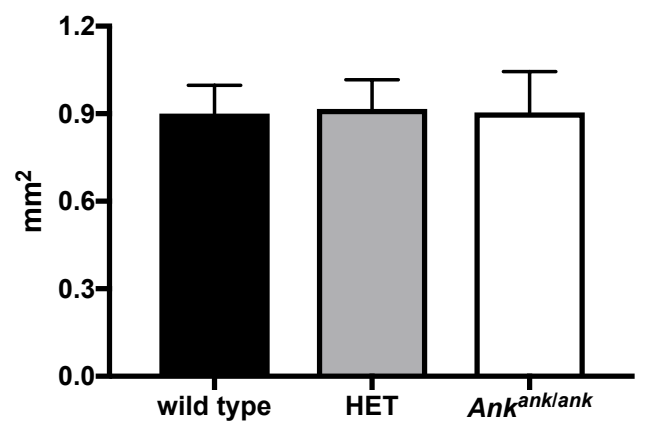

TMD

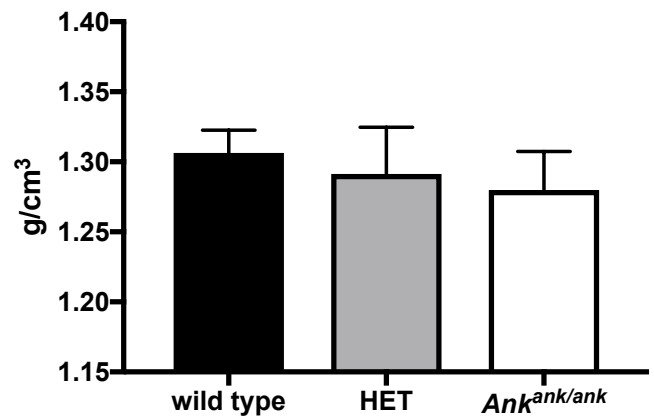

E

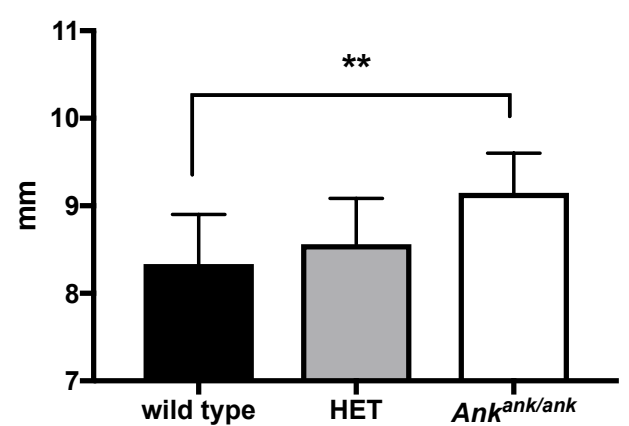

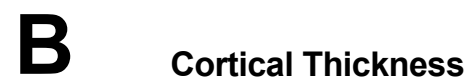

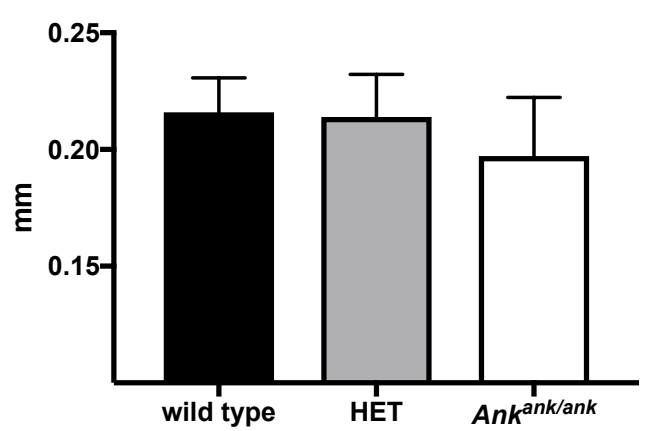

D

Bone Area Fraction

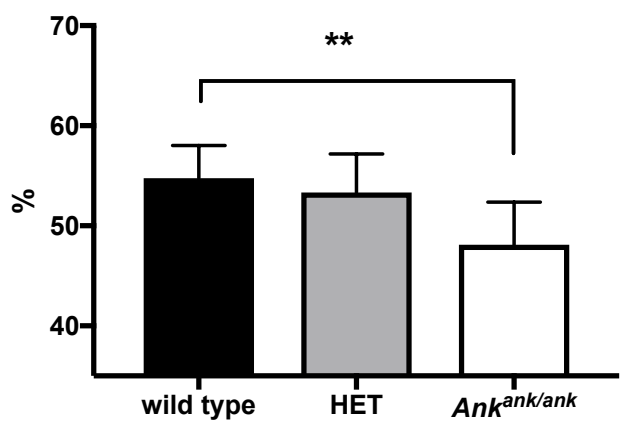

F

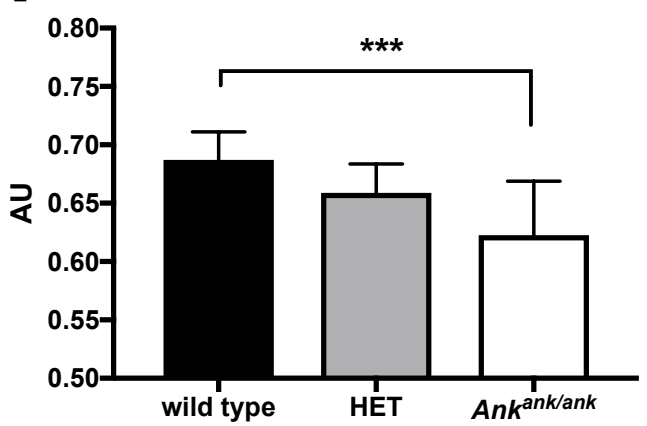

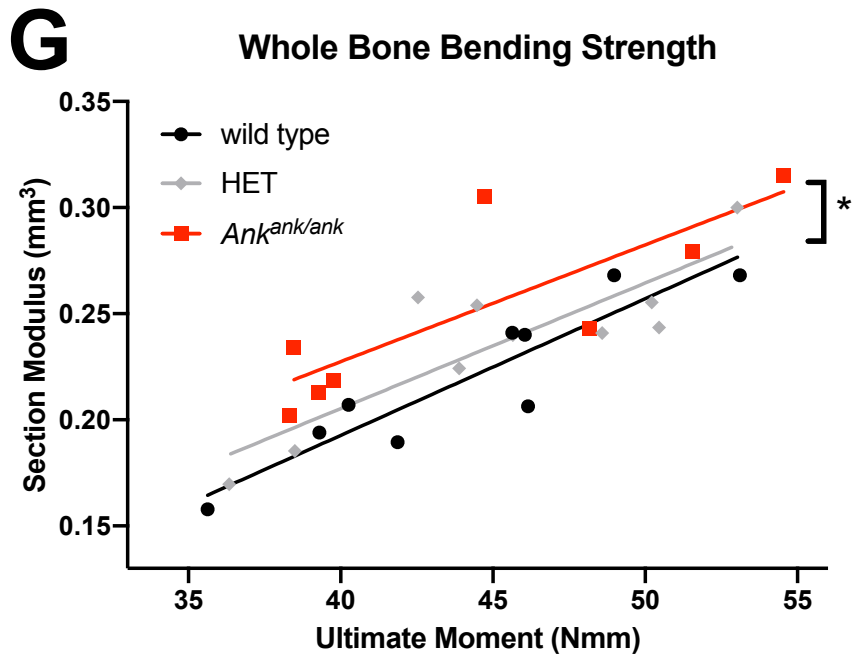

